\title{
Mutation landscape in patients with myelofibrosis receiving ruxolitinib or hydroxyurea
}

\author{
Annalisa Pacilli ${ }^{1}$, Giada Rotunno ${ }^{1}$, Carmela Mannarelli ${ }^{1}$, Tiziana Fanelli ${ }^{2}$, Alessandro Pancrazzi ${ }^{1}$, Elisa Contini ${ }^{1}$

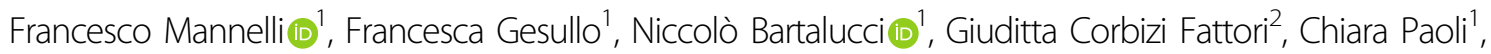 \\ Alessandro M. Vannucchi (iD ${ }^{1}$ and Paola Guglielmelli (i)
}

\begin{abstract}
Refractoriness to ruxolitinib in patients with myelofibrosis (MF) was associated with clonal evolution; however, whether genetic instability is promoted by ruxolitinib remains unsettled. We evaluated the mutation landscape in $71 \mathrm{MF}$ patients receiving ruxolitinib $(n=46)$ and hydroxyurea $(n=25)$ and correlated with response. A spleen volume response (SVR) was obtained in $57 \%$ and $12 \%$, respectively. Highly heterogenous patterns of mutation acquisition/loss and/or changes of variant allele frequency (VAF) were observed in the 2 patient groups without remarkable differences. In patients receiving ruxolitinib, driver mutation type and high-molecular risk profile (HMR) at baseline did not impact on response rate, while HMR and sole ASXL1 mutations predicted for SVR loss at 3 years. In patients with SVR, a decrease of $\geq 20 \%$ of JAK2V617F VAF predicted for SVR duration. VAF increase of non-driver mutations and clonal progression at follow-up correlated with SVR loss and treatment discontinuation, and clonal progression also predicted for shorter survival. These data indicate that (i) ruxolitinib does not appreciably promote clonal evolution compared with hydroxyurea, (ii) VAF increase of pre-existing and/or (ii) acquisition of new mutations while on treatment correlated with higher rate of discontinuation and/or death, and (iv) reduction of JAK2V617F VAF associated with SVR duration.
\end{abstract}

\section{Introduction}

Ruxolitinib is a JAK1 and JAK2 inhibitor approved for the treatment of intermediate and high-risk patients with primary (PMF) and post-polycythemia vera (PPV-MF) and post-essential thrombocythemia (PET-MF) myelofibrosis ${ }^{1,2}$. Long-term follow-up studies have confirmed its efficacy in inducing rapid improvements in splenomegaly, diseaseassociated symptomatology and overall quality of life $\mathrm{f}^{3}$, while the impact on overall survival ${ }^{3-7}$ remains debated $^{7-9}$. Furthermore, a true disease-modifying effect is questioned,

\footnotetext{
Correspondence: Alessandro M. Vannucchi (amvannucchi@unifi.it) ${ }^{1}$ CRIMM, Centro di Ricerca e Innovazione per le Malattie Mieloproliferative, Azienda Ospedaliera Universitaria Careggi, Dipartimento di Medicina Sperimentale e Clinica, Università degli Studi, Firenze, Italy

2Doctorate School GenOMeC, University of Siena, Siena, Italy These authors contributed equally: Alessandro M. Vannucchi, Paola Guglielmelli.
}

since only a minority of the patients experience significant molecular responses and reduction of bone marrow fibrosis $^{10,11}$. In spite of rapid, sometimes dramatic, clinical benefits, at least $50 \%$ of the patients become overtly refractory to ruxolitinib or experience progressively increase of spleen volume or reappearance of symptoms, necessitating soon or later discontinuation of therapy. In the COMFORT-I and COMFORT-II phase 3 trials, discontinuation due to loss of response, disease progression and treatment-related adverse events involved $\approx 50 \%$ of the patients at 3 years and $75 \%$ at 5 years ${ }^{12-15}$. Discontinuation of ruxolitinib because of loss of response was associated with dismal outcome among 107 patients enrolled in a phase $1 / 2$ study, with median survival after discontinuation of only 14 months ${ }^{16}$. Managing patients who fail ruxolitinib therapy may be challenging especially when stem cell transplantation is not feasible, and 
options include alternative JAK inhibitor therapy, alone or in combination, in the setting of clinical trials, novel agents, splenectomy, other palliative approaches ${ }^{17}$.

There is yet no mechanistic explanation for the development of resistance to ruxolitinib ${ }^{18}$. Advocated mechanisms include reactivation of JAK/STAT signaling by JAK heterodimer formation ${ }^{19}$, protective effects of cytokines $^{20,21}$, incomplete target inhibition by type I JAK inhibitor as is ruxolitinib ${ }^{21}$, while acquired activating $J A K 2$ mutations have been described in cell lines but not yet reported in patients ${ }^{22,23}$.

To date few studies have investigated the molecular variable that may be associated with response and durability of response to ruxolitinib. We reported that type of driver mutations and presence of HMR mutations at baseline, were indifferent as regards the obtainment of clinical responses (SVR and symptomatic improvement) at week 24 and week 48 in the COMFORT-II trial ${ }^{24}$. On the other hand, it was suggested that a JAK2V617F variant allele frequency (VAF) $>50 \%$ was associated with greater likelihood of $\mathrm{SVR}^{25}$. On the other hand, in a series of a long-term treated patients included in a phase $1 / 2$ trial and analyzed by NGS panel of 28 non-driver recurrently mutated genes, the number of non-driver mutations at baseline had an impact on SVR; patients with 2 or less mutations had nine-fold higher odds of achieving SVR than those with 3 or more mutations, who also had shorter time to discontinuation of therapy ${ }^{26}$. A shorter time to treatment failure was also noticed in a study of 100 patients, including 23 treated with momelotinib, in association with an HMR profile and presence of ASXL1 and $E Z H 2$ mutations $^{27}$. More recently, among 86 patients receiving ruxolitinib for a median of 79 months, clonal evolution, hallmarked by acquisition of new mutations under treatment, was associated with significantly shorter survival after therapy discontinuation compared to patients without clonal evolution ${ }^{16}$.

The purpose of our study was to analyze first, whether attainment and duration of clinical responses in patients with MF receiving ruxolitinib in a real-life setting was associated with unique mutation landscape at baseline and/or changes of mutation profile and VAF at follow-up, and whether clonal evolution might be attributed directly to selective pressure induced by ruxolitinib on preexisting clones and/or through the facilitation of emergence of new mutated clones, in comparison with standard therapy represented by hydroxyurea.

\section{Materials and methods Patients}

Seventy-one patients (42 with PMF, 29 with PPV/PETMF) in active follow-up at our Institution were included. Diagnosis of PMF fulfilled the 2016 revised World Health Organization (WHO) criteria $^{28,29}$ while criteria of the International Working group for Myelofibrosis Research and Treatment (IWG-MRT) were used for the diagnosis of PPV-MF and PET-MF ${ }^{30}$. Twenty-five patients (19 PMF, 6 PPV/PET-MF) were treated with hydroxyurea (HU) and 46 (23 PMF, 23 PPV/PET-MF) with ruxolitinib in a real-life setting. The study inclusion criteria for patients receiving ruxolitinib and $\mathrm{HU}$ were: (1) to have been treated continuously with the drug for at least 1 year and (2) to have stored a "baseline" blood sample (at the time of treatment start) and a "follow-up" sample collected at least one year later that, for patients who discontinued, was coincident with treatment discontinuation. Patients treated with HU were randomly selected from our database to match, in terms of diagnosis, clinical characteristics and follow-up criteria, the patients receiving ruxolitinib; all included patients had to be DIPSS intermediate-2/high risk and have a baseline spleen that was palpable at $>5 \mathrm{~cm}$ from the left costal margin (LCM). The dose of $\mathrm{HU}$ was according to clinical practice and according to the label for ruxolitinib; both drugs were titrated depending on clinical and hematologic criteria and toxicity. There was no hold of treatment in either group during the study period. All patients provided written informed consent to participate to the study, sponsored by AGIMM (AIRCGruppo Italiano Malattie Mieloproliferative) and supported by MYNERVA (Myeloid Neoplasms Research Venture-AIRC) project; the study was approved by local Ethical Committee.

\section{Definitions of clinical response and outcome}

Response of symptoms or splenomegaly was according to the IWG-MRT/ELN criteria $^{31}$. A spleen response was adjudicated in case a spleen extending 5 to $10 \mathrm{~cm}$ from the LCM at baseline became no palpable or a spleen that was $>10 \mathrm{~cm}$ decreased by $\geq 50 \%$. A symptoms response was considered in case of a $>50 \%$ reduction of the MPN-SAF Total Symptom Score (MPN-SAF TSS) ${ }^{32}$. Time to clinical response and to response loss was calculated from baseline to the date of achieving or loosing, respectively, the above criteria of spleen and/or symptoms response. Overall survival was calculated from the first day of treatment to the last follow-up or death. Time to discontinuation was calculated from the date of treatment start to the date of therapy discontinuation.

\section{Methods}

All patients were annotated for driver mutations and an additional panel of 24 "myeloid" genes by Next Generation Sequencing (NGS). Mutational analysis was performed on high-quality DNA obtained from density gradient-purified granulocytes from peripheral blood. Samples were collected before starting HU/ruxolitinib therapy (baseline) and at different time points thereafter; 
the last available sample ("follow-up" -FU- sample for the purpose of this study) was collected at least one year later for patients who were still receiving the drug (in case of patients with sustained response), or at therapy discontinuation for patients who discontinued because of no response/loss of response.

$J A K 2 \mathrm{~V} 617 \mathrm{~F}$ and $M P L$ W515 $\mathrm{L} / \mathrm{K}$ mutations were detected by real time (RT)-qPCR ${ }^{33,34}$ and high-resolution melting analysis (HRMA) ${ }^{35}$ followed by bidirectional Sanger sequencing ${ }^{36}$, respectively. CALR mutations were identified by bidirectional sequencing and capillary electrophoresis (CE) and classified as type 1/like or type $2 /$ like, as described ${ }^{37,38}$. CALR amplification was carried out with a 6-FAM-labeled forward primer followed by fragment analysis on a ABI Prism 310 Genetic Analyser (GeneMapper Software 4.1; Applied Biosystems, Forest City, CA, USA). All samples with an additional peak to the wild-type one were further evaluated by direct Sanger sequencing. The level of detection was $<0.1 \%$ for JAK2V617F mutations and 1\% for MPLW515 mutations using RT-qPCR and HRMA, respectively, and $1 \%$ by capillary electrophoresis for CALR mutations.

High molecular risk mutations (HMR; ASXL1, EZH2, $S R S F 2, I D H 1, I D H 2)$ and other myeloid-neoplasm associated gene mutations (CBL, C-KIT, CSF3R, CUX1, DNMT3A, ETNK1, IKZF1, KRAS, NFE2, NRAS, PTPN11, RUNX1, SETBP1, SH2B3, SF3B1, TET2, TP53, U2AF1, ZRSR2), as well as the entire coding regions of JAK2 and MPL, were evaluated by using a custom panel for NGS on Ion Torrent PGM platform (ThermoFisher Scientific, Waltham, Massachussets, USA). NGS raw reads were aligned against the GRCh38/hg38 using NextGENe ${ }^{\circledR}$ software 2.4.2 for variants call with a variant allele frequency (VAF) threshold of $\geq 2 \%$, in case of previously unreported mutations, and $\geq 1 \%$ for known hotspots, and depth of coverage of at least $100 \times$ (SoftGenetics, LLC, State College, PA). Mutations in the exonic regions were filtered by available databases (dbSNP, COSMIC, 1000 genome, ExAC); protein function predictor algorithms (Polyphen2, SIFT, MutationTester, FATHMM, Gerp) were used to predict functional relevance of mutations, and only indels and pathogenetic variants were considered. An HMR category was defined by the presence of $\geq 1$ of HMR genes mutations; patients lacking these mutations were defined at "low molecular risk" (LMR). For the analysis, we recorded any modification of VAF, either decreasing or increasing, that had a magnitude of at least $20 \%$ compared to baseline. Mutations were defined "acquired" in case of de novo detection ( $\geq 2 \%$ VAF) and "lost" in case the VAF of a mutation detected became less than $0.1 \%$.

\section{Statistical analysis}

Best overall response of splenomegaly was adjudicated at any time while the patients was on continuous drug administration using the IWG-MRT/ELN revised criteria $^{31}$; assessment at 24 and 48 weeks was also performed, as in the COMFORT trials. Response of symptoms was annotated at any time point between baseline and 48 weeks. Categorical variables were compared using $\chi^{2}$ or Fisher's exact test and continuous variables were compared using Mann-Whitney or Kruskal-Wallis tests. Comparison of response to ruxolitinib across molecular categories was tested for homogeneity of distributions using chi-square test. Survival time estimates, including survival curves by response status, were obtained with the Kaplan-Meier method; the hazard ratio (HR) was determined using a Cox proportional hazards model. All $P$ values are 2-tailed and were considered significant when $P<.05$. Statistical analyses were performed using SPSS v.25 (IBM).

\section{Results}

\section{Patients' characteristics at baseline}

Patients' characteristics at baseline are reported in Table 1; forty-six patients received ruxolitinib (ruxo-patients) and 25 patients received hydroxyurea (HU-patients). There was no statistically significant difference between the two groups regarding main clinical and laboratory parameters (Table 1), a part for a greater proportion of patients with larger ( $>10 \mathrm{~cm}$ from LCM) splenomegaly among the ruxo-patients compared to HU-patients (76.1\% vs. $40.0 \% ; P<.0001)$. The median follow-up duration from the initiation of therapy was 3.4 years (range, 1.0-7.4; $P=.981$ ) in patients receiving ruxolitinib and 2.9 years (range, 1.0-11.1) in HU-patients. The proportion of study patients who had a follow-up sample collected at $>5$ years of treatment was $36.0 \%$ and $34.8 \%$ for ruxolitinib and $\mathrm{HU}$, respectively $(P=.897)$. Over the study period, 11 of $46(23.9 \% \%)$ ruxo-patients discontinued therapy for loss of response, 1 patient $(2.2 \%)$ progressed to acute leukemia and 20 patients (43.5\%) died; fifteen patients $(32.6 \%)$ were still receiving ruxolitinib at latest follow-up. In the HU group, 10 patients (40.0\%) died during the follow-up, one (4.0\%) of which had progressed to acute leukemia, while $15(60 \%)$ were still on therapy.

\section{Mutation landscape at baseline}

The mutation landscape of patients at baseline is shown in Fig. 1. A JAK2V617F mutation was found in 54 patients (76.0\% of total; $82.6 \%$ of ruxo-patients and $64 \%$ of HUpatients), CALR mutation in 12 (16.9\%; $13 \%$ of ruxopatients and $24 \%$ of HU-patients), of which 10 (83\%) were Type 1 and 2 (17\%) Type 2, MPL mutations in 3 patients ( 1 in the ruxo-group and 2 in the HU-group); 1 patient in each cohort was triple negative. Non-driver mutations represented at $>5 \%$ in the series were ASXL1 (36.6\%; $41.3 \%$ of ruxo-patients and $28.0 \%$ of HU-patients), TET2 
Table 1 Baseline clinical and hematologic characteristics of study patients stratified according to the treatment

\begin{tabular}{|c|c|c|c|}
\hline Variables & Ruxo-patients $(N=46)$ & HU-patients $(N=25)$ & $P$ \\
\hline \multicolumn{4}{|l|}{ Diagnosis, n.(\%) } \\
\hline PMF & $23(50.0)$ & $19(76.0)$ & \multirow[t]{3}{*}{.050} \\
\hline PPV-MF & $16(35.0)$ & $2(8.0)$ & \\
\hline PET-MF & $7(15.0)$ & $4(16.0)$ & \\
\hline Follow-up from the start of treatment, $y$ : median (range) & $3.4(1.0-7.4)$ & $2.9(1.0-11.1)$ & .981 \\
\hline Time from diagnosis to treatment & $1.1(0.3 .6)$ & $2.8(0-3.7)$ & .743 \\
\hline Males, $n(\%)$ & $22(48.0)$ & $15(60.0)$ & .232 \\
\hline Age, y; median (range) & $63.4(35.0-81.0)$ & $66.9(43.0-88.0)$ & .087 \\
\hline Hemoglobin, g/L; median (range) & $107(70-140)$ & $99(89-109)$ & .912 \\
\hline Leukocytes, $\times 10^{9} / \mathrm{L}$; median (range) & $13.3(3.0-103.0)$ & $11.8(5.8-14.0)$ & .917 \\
\hline Platelets, $\times 10^{9} / L$; median (range) & $333(52-750)$ & $433(227-1378)$ & .090 \\
\hline Circulating blasts $\geq 1 \% ; n(\%)$ & $12(26.1)$ & $7(28.0)$ & .993 \\
\hline Constitutional symptoms; $n$ (\%) & $43(93.5)$ & $21(84.0)$ & .558 \\
\hline Splenomegaly > 10 cm from LCM; $n(\%)$ & $35(76.1)$ & $10(40.0)$ & $<.0001$ \\
\hline Patients with cytogenetic information; $N=$ (\% of total) & $42(91.3)$ & $21(84.0)$ & .202 \\
\hline Abnormal cytogenetics & $19(45.2)$ & $6(28.6)$ & \\
\hline Unfavorable karyotype & $4(9.5)$ & $2(9.5)$ & \\
\hline \multicolumn{4}{|l|}{ DIPSS } \\
\hline Intermediate-2 & $40(86.9)$ & $20(80.0)$ & \multirow[t]{2}{*}{.441} \\
\hline High & $6(13.1)$ & $5(20.0)$ & \\
\hline
\end{tabular}

Note: Unfavorable karyotype indicates any of the following: $+8,-7 / 7 q-$, i(17q), inv(3), $-5 / 5 q, 12 p-$, or $11 q 23$ rearrangements. DIPSS, Dynamic International Prognostic Scoring System. DIPSS uses five independent predictors of inferior survival: age $>65$ years, hemoglobin $<10 \mathrm{~g} / \mathrm{dL}$, leukocytes $>25 \times 10^{9} / \mathrm{L}$, circulating blasts $\geq 1 \%$, constitutional symptoms, resulting in four (low, intermediate-1, intermediate-2 and high) risk categories

(22.5\%; $21.7 \%$ of ruxo-patients and $24.0 \%$ of HU-patients), NFE2 $(12.6 ; 15.2 \%$ of ruxo-patients and $8.0 \%$ of $\mathrm{HU}$ patients), ZRSR2 (11.7\%; $11.6 \%$ of ruxo-patients and $11.8 \%$ of HU-patients), EZH2 (8.5\%; 6.5\% of ruxo-patients and $12.0 \%$ of HU-patients), SF3B1 (7.0\%; $2.2 \%$ of ruxo-patients and $16.0 \%$ of HU-patients), $S H 2 B 3(7.0 \%$; $8.7 \%$ of ruxopatients and $4.0 \%$ of HU-patients). Mutations of TP53 were found in 3 patients (4.2\%), 2 of whom were in the HU-group. No mutation was found in CBL, C-KIT, CSF3R, CUX1, DNMT3A, IKZF1, RUNX1, IDH2. Considering all non-driver mutated genes evaluated, a total of 54 patients $(76 \%)$ showed at least one somatic variant: 36 in the ruxo-group (78.3\%) and $18(72.0 \%)$ in the HUgroup; 2 or more mutations were found in 19 ruxopatients $(41.3 \%)$ and $9 \mathrm{HU}$-patients $(36.0 \%)$; 3 or more mutations were harbored by 4 ruxo-patients $(8.7 \%)$ and 2 HU-patients (8.0\%). A total of 30 patients (42.2\%) were considered at high-molecular risk (HMR), including 21 in the ruxo-group (45.6\%) and 9 (36.0\%) in the HU group; 2 or more HMR mutations were found in 5 patients $(7.0 \%)$, of which 2 in the ruxo-group (8.0\%) and 3 in HU-patients (12\%). With the limitation of small numbers, we compared rate of non-driver mutations in patients with PMF vs. PPV/PET MF. A total of $78.6 \%$ of PMF vs. $72 \%$ of $\mathrm{PPV} / \mathrm{PET}$ MF patients had $\geq 1$ mutation $(p=0.58)$, and $40.5 \%$ of PMF vs. $44.8 \%$ of PPV/PET-MF were $\mathrm{HMR}^{+}$.

\section{Mutation landscape at follow-up sample}

In the follow-up sample of patients receiving ruxolitinib, a change, either increase or decrease (see Materials and Methods for definition) of the VAF of any mutation (driver and non-driver) detected at baseline was detected in $41.3 \%$ and $34.8 \%$, respectively (Fig. 1, panel A). Concerning driver mutations (Fig. 2, panel A), the $J A K 2 \mathrm{~V} 617 \mathrm{~F}$ VAF overall decreased from $83.4 \pm 19.3 \%$ to $78.8 \pm 25.3 \%$ $(P=.43)$ : in detail, the VAF increased in 6 patients $(15 \%$; median increase $+27 \%$, range $+20 \%$ to $+50 \%$ ) and decreased in 9 (23\%; median $-39 \%$, range -21 to $-62 \%$ ); CALR VAF showed a slight, not significant increase from $44.7 \pm 10.6 \%$ to $48.7 \pm 10.3 \% \quad(P=.52)$ : in detail it increased by $32 \%$ in 1 patient and remained unchanged in 5 patients; in the one MPL mutated patient, the VAF decreased from 37 to $14 \%(-38 \%)$. As regards non-driver mutations (Fig. 1, panel A), the VAF overall increased in 


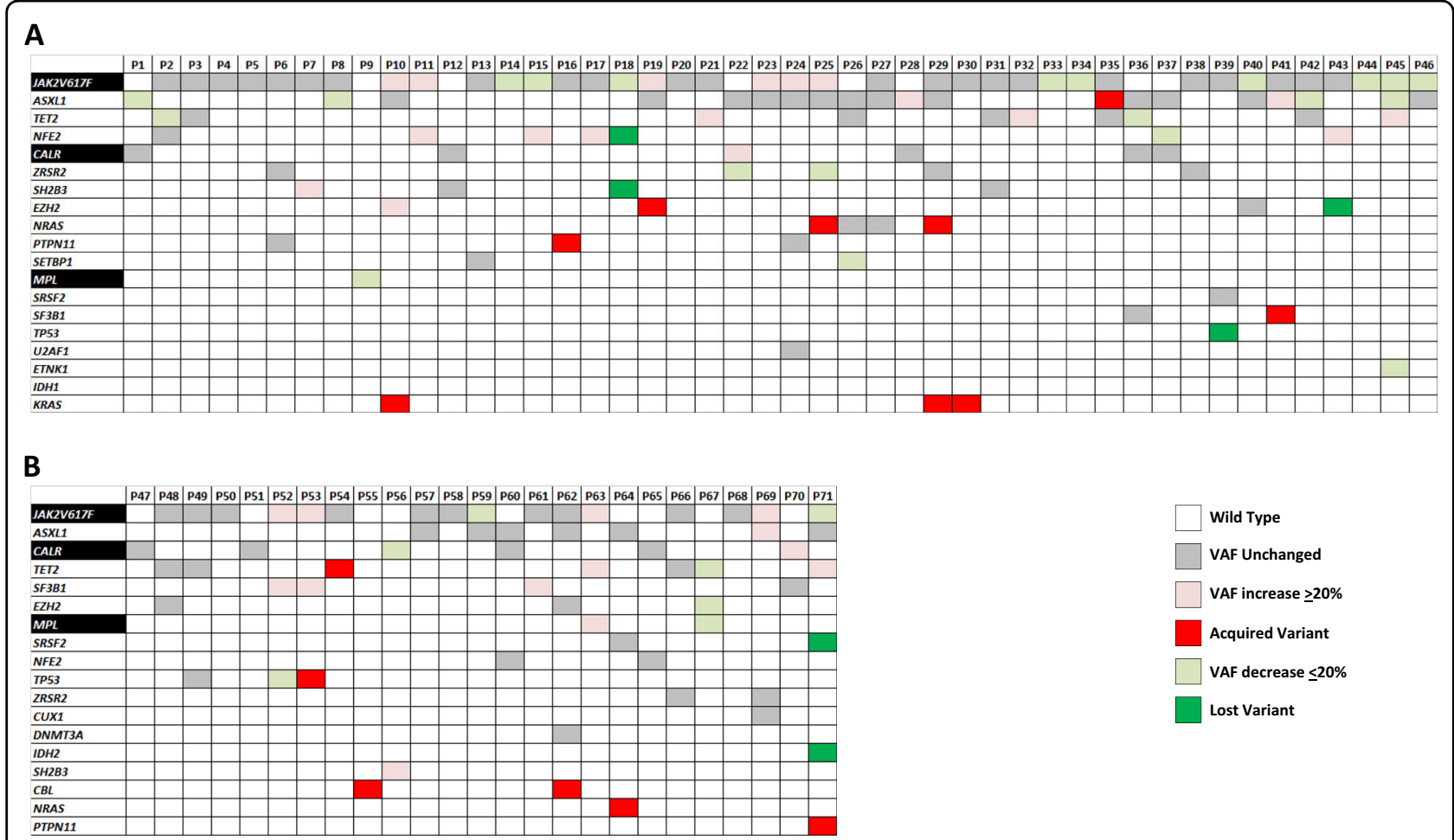

Fig. 1 Landscape plot of mutations in the study population. Each column represents an individual patient. a: ruxolitinib treated patients; b: HUtreated patients. color code: gray indicates a mutation detected at baseline that remained unchanged at the latest follow-up sample; pink colour indicates a mutation whose VAF increased of at least 20\% compared to baseline; red colour indicates a newly acquired mutation at follow-up; light green colour indicates a mutation whose VAF decreased of at least $20 \%$ compared to baseline; green colour indicates a mutation that, while detected at baseline, was no longer detected in the follow-up sample

the follow-up sample in 11 patients $(24 \%$ of all mutated patients) by a median value of $104 \%$, ranging from $+20 \%$ to $+480 \%$, compared to baseline one. The involved genes were NFE2 (number of variants =4), TET2 (number of variants $=3$ ), $A S X L 1$ (number of variants $=2$ ), $S H 2 B 3$ and $E Z H 2$ (one variant each). On the contrary, the VAF decreased in 10 patients $(22 \%)$ by a median of $30 \%(-23 \%$ to $-69 \%$ ); the involved genes were ASXL1 (number of variants $=4), T E T 2$ and $Z R S R 2$ (2 variants each), NFE2, SETBP1, and ETNK1 (1 variant each). Four mutations detected at baseline in 3 patients (6.5\%; Fig. 1, panel A), involving EZH2, NFE2, SH2B3, and TP53, were no longer detected in the follow-up sample. Conversely, acquisition of a new mutation was observed in 8 cases (17.4\%; Fig. 1, panel A), involving EZH2, SF3B1, ASXL1, PTPN11 (one each), NRAS (2 cases), and KRAS (3 cases), in one case concurrently with acquisition of a NRAS mutation. Of note, acquisition of novel mutations was observed only in $J A K 2 \mathrm{~V} 617 \mathrm{~F}$ mutated patients compared to no CALR mutated patients.

Among patients receiving $\mathrm{HU}$, an increase or a decrease of the VAF of any baseline mutation (driver and nondriver) was detected in $20.8 \%$ and $33.3 \%$, respectively (Fig. 1, panel B). Concerning the driver mutations (Fig. 2, panel
B), the JAK2V617F VAF overall increased from $54.2 \pm$ $20.3 \%$ to $60.5 \pm 22.7 \%(\mathrm{P}=.59)$, in detail, the VAF increased in 4 patients ( $16 \%$, range $+33 \%$ to $+88 \%$ ) and decreased in 2 patients by $41 \%$ and $63 \%$, respectively. In $C A L R$ and MPL mutated patients the VAF increased in 1 case and decreased in 1 case each (Fig. 2, panel B). As regards non-driver mutation (Fig. 1, panel B), the VAF overall increased in 7 patients $(28 \%$ of all mutated patients) by a median $+94.9 \%$ (range $+22.8 \%$ to $+174 \%$ ) in the follow-up sample compared to baseline one; the involved genes were SF3B1 and TET2 (number of variants = 2), ASXL1 and SH2B3, (1 variant each). On the contrary, the VAF decreased in 2 patients $(8 \%)$, the genes involved were TP53 $(-65 \%)$ and TET2 $(-50 \%)$. One patient showed disappearance of baseline mutation in SRSF2 and IDH1 (VAF of $6.3 \%$ and $8.2 \%$, respectively), while acquisition of new mutation at follow-up sample occurred in 6 patients (24\%); involved genes were TET2, TP53, NRAS, PTPN11 and CBL (in 2 patients) (Fig. 1, panel B). Acquisition of novel mutation was observed in four $J A K 2 \mathrm{~V} 617 \mathrm{~F}$ mutated patients and in 2 triple negative patients, compared to no patients with CALR mutation.

Clonal progression occurred in $21.4 \%$ of PMF vs. $17.2 \%$ of PPV/PET MF $(P=.66)$. 


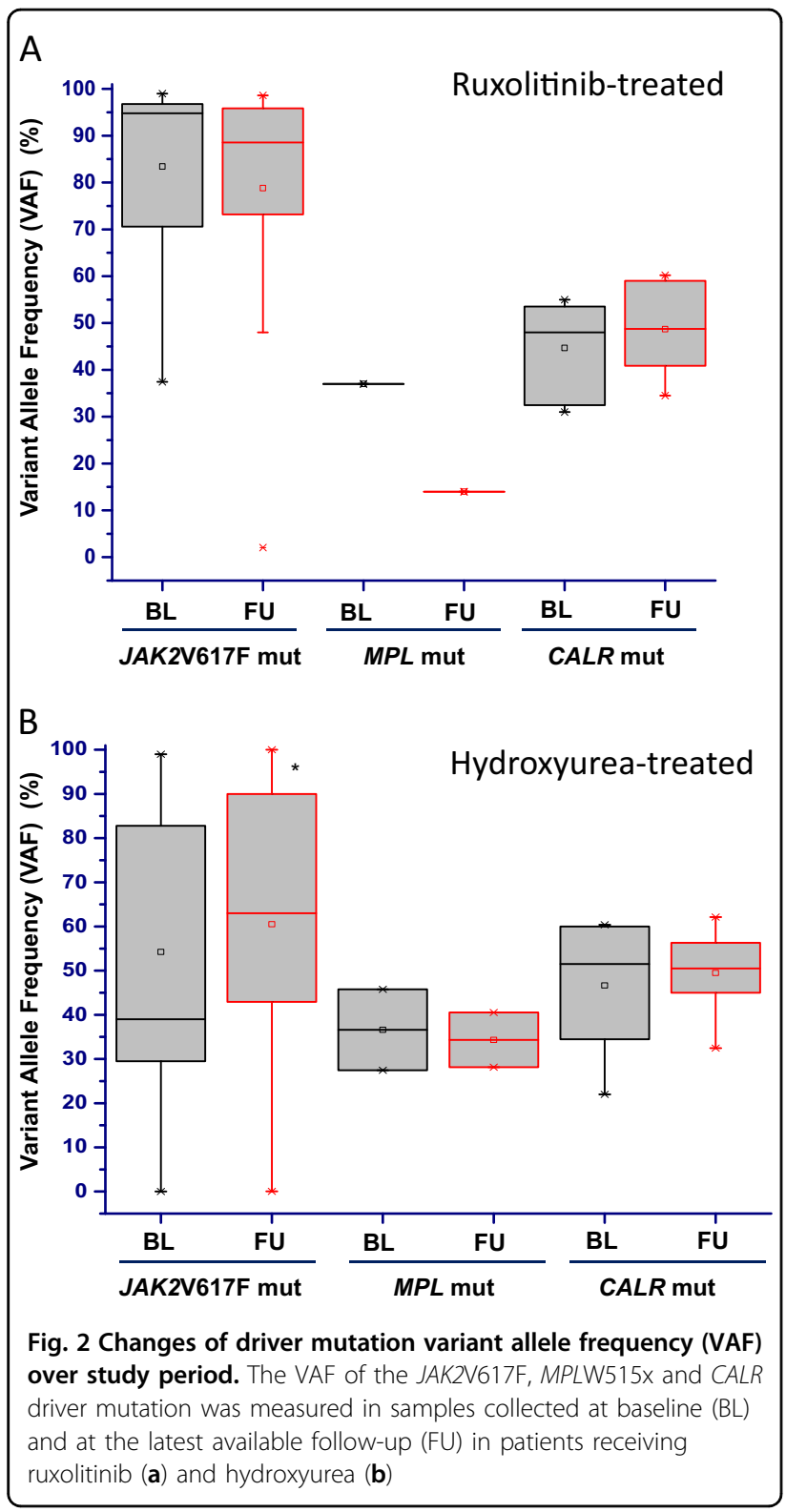

\section{Correlation of mutation landscape at baseline with clinical response}

Among patients receiving ruxolitinib, response of symptoms and splenomegaly (by IWG-MRT/ELN criteria $^{31}$ ) was achieved by 78 and $57 \%$ of the patients after a median of 2.0 months (range, 1-37 months) and 5.8 months, respectively (range, 1-49 months); of these, $11(31 \%)$ and $12(30.8 \%)$ patients lost clinical response after a median of 2.4 years (range, 0.5-5.2) for symptoms and 1.8 years for SVR (range, 0.7-5.0). Since in the HU group only $1(4 \%)$ and $3(12 \%)$ patients had achieved symptoms and spleen response, respectively, at any time during follow-up, further analysis was restricted to patients treated with ruxolitinib.
We found no difference in the proportion of patients with $J A K 2$ V617F mutation vs. patients with CALR mutation who achieved either a spleen or symptom response, confirming previous reports; a SVR and/or a symptom response was obtained by $54.1 \%$ and $86.0 \%$ of $J A K 2 \mathrm{~V} 617 \mathrm{~F}$ mutated patients compared to $66.7 \%$ and $60.0 \%$ of $C A L R$ mutated patients. There was also no difference in terms of SVR and/or a symptom response among patients with a $J A K 2$ V617F VAF greater or lower than $50 \%$.

Presence of an HMR status at baseline did not affect the likelihood of obtaining SVR or symptom response, as we previously reported ${ }^{24}$. A spleen response was obtained by $42.9 \%$ of HMR patients compared to $44.0 \%$ of the LMR counterpart at 24 weeks, and $42.8 \%$ compared to $56.0 \%$ at 48 weeks; the best overall spleen response rate was $51 \%$ and $64 \%$, respectively. The rate of symptoms response was $68.4 \%$ in HMR patients compared to $64.0 \%$ in un-mutated patients at 24 weeks, and $73.7 \%$ compared to $76.0 \%$ at 48 weeks. On the other hand, baseline HMR status was significantly associated with loss of SVR, with a HR of 3.6 (95\%CI, 12.0-16.7; $P=.005$ ) compared to LMR patients (Fig. 3, panel A); at 3 years, 14\% of LMR patients had lost spleen response compared to $46 \%$ of the HMR category $(P=.005)$. There was no difference in rate of symptom response depending on HMR vs. LMR status.

We also analyzed the impact of sole $A S X L 1$ mutations at baseline, the most frequently mutated gene, on response. Presence of $A S X L 1$ mutation at baseline did not impact on the rate of spleen or symptom response. A spleen response was obtained by $47.4 \%$ of ASXL1- mutated patients compared to $40.7 \%$ of the un-mutated counterpart at 24 weeks, and $47.0 \%$ compared to $51.9 \%$ at 48 weeks. Symptoms response was achieved by $64.7 \%$ of ASXL1 mutated patients compared to $66.7 \%$ at 24 weeks, and $70.6 \%$ compared to $77.8 \%$ at 48 weeks. Furthermore, presence of sole $A S X L 1$ mutation was associated with a significantly shorter duration of spleen volume reduction; the probability of maintaining a spleen response at 3 years among ASXL1 mutated patients was 33\% compared to $87 \%$ of un-mutated patients $(P=.009)$.

\section{Impact of clonal evolution at follow-up on clinical response}

A decrease of the $J A K 2 \mathrm{~V} 617 \mathrm{~F}$ VAF at any time point during treatment was significantly associated with maintenance of SVR $(P=.015)$; in fact, none of the 7 patients who showed decrease of $\geq 20 \%$ from baseline $J A K 2 \mathrm{~V} 617 \mathrm{~F}$ VAF lost SVR compared to 6 out of 13 (46.1\%) who showed stable or increased $J A K 2 \mathrm{~V} 617 \mathrm{~F}$ VAF $(\mathrm{HR}=61.8$, 95\% CI 1.01-870.2; Fig. 3, panel B). Similar analysis could not be done for $C A L R$ mutated patients, owing that there was no significant modification of CALR VAF during follow-up.

We found that loss of SVR was significantly associated with clonal progression, ie the acquisition of $\geq 1$ novel 


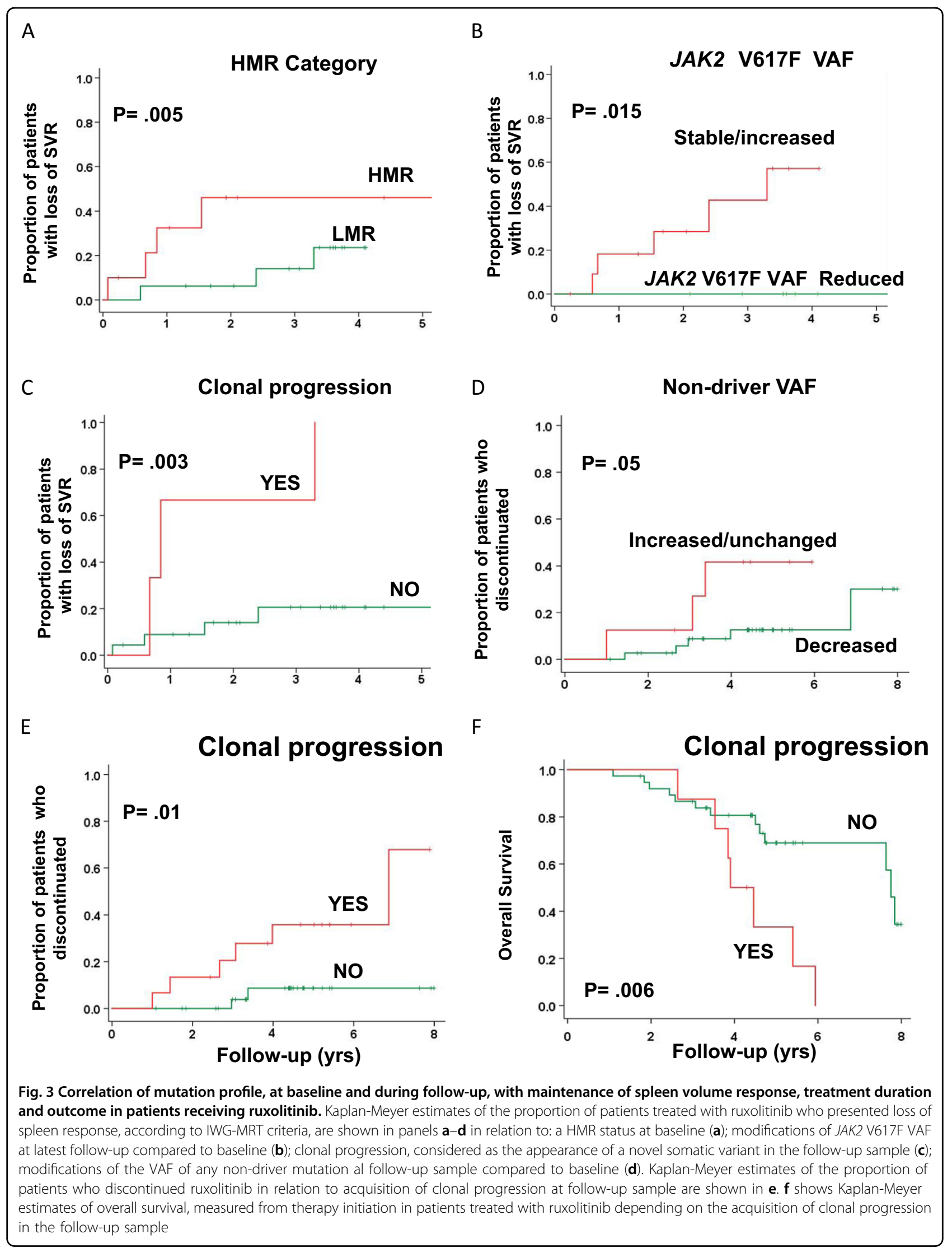


mutation in any non-driver genes during follow-up; all patients with SVR loss during follow-up had evidence of clonal progression compared to $21 \%$ who lost SVR without having clonal progression $(P=.006)$. Patients with clonal progression had median duration of SVR of 10 months (range, 8.4-13.0 month) compared to notreached in patients without clonal progression (HR 7.2, 95\% CI, 1.6-33.0; $P=.003$ ) (Fig. 3, panel C). A greater rate of therapy discontinuation due to SVR loss was found in patients showing VAF increase of any baseline mutation; at 4 years of treatment, the proportion of patients who discontinued ruxolitinib was $36 \%$ among those with VAF increase compared to $9 \%$ in those where VAF remained stable or decreased (HR 6.1, 95\% CI 1.2-30.5; P $=.01$; Fig. 3 , panel D). Clonal progression was also associated with a higher rate of treatment discontinuation (38\% vs. $13 \%$ for those without clonal progression; $P$ $=.05)$, accounting for an HR of 3.9 (95\%CI, 1.1-17.3; $P$ $=.01)$ (Fig. 3 panel E).

We did not find any impact of modifications of VAF of $J A K 2 \mathrm{~V} 617 \mathrm{~F}$ and other non-driver mutations on survival whilst acquisition of clonal progression at any study time point was associated with shorter overall survival; the median OS was 3.9 years (range, 3.1-4.6) compared to 7.7 years (7.5-8.0) for patients without clonal progression ( $\mathrm{HR}=3.6,95 \% \mathrm{CI} 1.4-9.7 ; P=.006$; Fig. 3 panel F). As many as $87.5 \%$ of patients with clonal progression documented at any study time point died compared to $34 \%$ of those without clonal progression $(P=.006)$.

\section{Discussion}

Results of current study confirm and extend previous reports on the impact of driver ${ }^{39,40}$ and non-driver ${ }^{24,26,27}$ baseline mutations, and of mutations acquired while on treatment (clonal progression) ${ }^{16}$, on response to treatment and response duration in patients with myelofibrosis receiving ruxolitinib. For the first time, our study included a control group of matched patients treated with hydroxyurea, that highlighted that MF is hallmarked by highly dynamic mutation landscape that is largely treatmentindependent, since modifications of mutation profile during follow-up were substantially similar in patients receiving ruxolitinib or hydroxyurea. These findings, while indicating that clonal progression is not facilitated by ruxolitinib itself but is intrinsically associated to the disease, may have practical relevance as concerns safety issues of ruxolitinib, particularly in the light of recent demonstration of aggressive B-cell lymphomas developing in ruxo-treated patients, that were shown to stem from Bcell clones pre-existing in the bone marrow ${ }^{41}$. We acknowledge that one limitation of the study is the limited number of patients in the hydroxyurea group, that might warrant confirmation in larger series. Of note, progression to PPV-MF and to acute leukemia in PV and MF patients receiving ruxolitinib was not increased compared to controls $^{3,4,14}$. However, acquisition of new mutations while on ruxolitinib has relevant clinical correlates, since it was associated with higher rate of discontinuation due to resistance to treatment and death.

Ruxolitinib has limited effects on JAK2V617F VAF in both $\mathrm{MF}^{10,15}$ and $\mathrm{PV}^{42}$, although some patients may present sustained decrease, irrespective of initial level ${ }^{10}$; complete molecular remissions are exceptional ${ }^{43}$. Shortterm SVR occurs independent of changes in JAK2V617F $\mathrm{VAF}^{15}$; however, we report the novel observation that patients with $J A K 2 \mathrm{~V} 617 \mathrm{~F}$ VAF reductions $\geq 20 \%$ from baseline have significantly greater likelihood to maintain sustained SVR. On the other hand, unlike previous report $^{25}$, we observed no impact of baseline JAK2V617F VAF on SVR. An additional finding, that deserves validation in larger series, was that clonal progression virtually segregated with patients harboring JAK2V617F mutation, and was not observed in CALR mutated patients. This may have to do with the increased genomic instability that characterizes cells expressing mutated JAK ${ }^{44}$, showing enhanced frequency of spontaneous homologous recombination events, DNA double strand breaks ${ }^{45}$, high levels of NHE- $1^{46}$ and deamidate Bcl- $\mathrm{x}_{\mathrm{L}}{ }^{47}$, that are possibly mediated by increased reactive oxygen species $(\mathrm{ROS})^{48,49}$.

Overall, findings from this study add novel information on the impact of baseline and on-treatment acquired genomic alterations on the clinical response in patients with MF receiving ruxolitinib, further highlighting the complexity of genomic landscape of these patients. The ultimate goal of this kind of studies is to identify a molecular profile at baseline that might guide decision regarding initiation of therapy with ruxolitinib; ${ }^{50}$ although presence of some molecular assets at baseline argue against a long-term likelihood to maintain SVR (current study and ${ }^{16,24,26,27}$ ), the large majority of patients obtain rapid clinical improvements, that are not otherwise achieved with other agents, making use of ruxolitinib a reasonable upfront strategy in symptomatic patients. Conversely, detection of specific molecular assets might be useful in counseling for an earlier shift from ruxolitinib to experimental therapies and/or stem cell transplantation. Probably the most relevant variable impacting on long-term maintenance of SVR to ruxolitinib, as well as on survival in patients who discontinue ${ }^{16}$, is the development of clonal progression while on treatment; serial assessment of mutation profile would be required to define whether the individual has manifested or not clonal progression, that is practically cumbersome ${ }^{50}$. All together, these particular observations might advocate for the prospective use of extensive genotyping in patients receiving ruxolitinib; however, we believe that further information are needed before this approach may be 
recommended in daily practice, also considering that alternative therapeutic options to ruxolitinib, outside clinical trials, in non-transplant eligible patients are very few and largely unsatisfactory ${ }^{17}$.

\section{Acknowledgements}

This work was supported by AIRC 5x1000 call "Metastatic disease: the key unmet need in oncology" to MYNERVA project, \#21267 (MYeloid NEoplasms Research Venture AIRC). A detailed description of the MYNERVA project is available at http://www.progettoagimm.it. Supported also by AIRC Investigator Grant 2014 \#15967 and Progetto Ministero della Salute grant No. GR-201102352109 to PG and by Regione Toscana, ITT project 2013, CUP: B16D14001130002

\section{Conflict of interest}

A.M.V. was involved in clinical trials with ruxolitinib, participated to advisory boards and speaker bureau for Novartis, and received institutional research grants from Novartis. All remaining authors declare no conflict of interest.

\section{Publisher's note}

Springer Nature remains neutral with regard to jurisdictional claims in published maps and institutional affiliations.

Received: 11 September 2018 Revised: 27 September 2018 Accepted: 15 October 2018

Published online: 22 November 2018

\section{References}

1. Verstovsek, S. et al. A double-blind, placebo-controlled trial of ruxolitinib for myelofibrosis. N. Engl. J. Med. 366, 799-807 (2012).

2. Harrison, C. et al. JAK inhibition with ruxolitinib versus best available therapy for myelofibrosis. N. Engl. J. Med. 366, 787-798 (2012).

3. Verstovsek, S. et al. Long-term survival in patients treated with ruxolitinib for myelofibrosis: COMFORT-I and -II pooled analyses. J. Hematol. Oncol. 10, 156 (2017).

4. Vannucchi, A. M. et al. A pooled analysis of overall survival in COMFORT-I and COMFORT-II, 2 randomized phase 3 trials of ruxolitinib for the treatment of myelofibrosis. Haematologica 100, 1139-1145 (2015).

5. Harrison, C. N. et al. Long-term findings from COMFORT-\|l, a phase 3 study of ruxolitinib vs best available therapy for myelofibrosis. Leukemia 31, 775 (2017).

6. Cervantes, F. \& Pereira, A. Does ruxolitinib prolong the survival of patients with myelofibrosis? Blood 129, 832-837 (2017).

7. Passamonti, F. \& Maffioli, M. The role of JAK2 inhibitors in MPN seven years after approval. Blood 131, 2426-2435 (2018).

8. Barbui, T. et al. Philadelphia chromosome-negative classical myeloproliferative neoplasms: revised management recommendations from European LeukemiaNet. Leukemia 32, 1057-1069 (2018).

9. Marchetti, M. et al. Which patients with myelofibrosis should receive ruxolitinib therapy? ELN-SIE evidence-based recommendations. Leukemia 31, 882-888 (2017).

10. Deininger, M. et al. The effect of long-term ruxolitinib treatment on JAK2p. V617F allele burden in patients with myelofibrosis. Blood 126, 1551-1554 (2015).

11. Kvasnicka, H. M. et al. Long-term effects of ruxolitinib versus best available therapy on bone marrow fibrosis in patients with myelofibrosis. J. Hematol. Oncol. 11, 42 (2018).

12. Verstovsek, S. et al. Three-year efficacy, overall survival, and safety of ruxolitinib therapy in patients with myelofibrosis from the COMFORT-I study. Haematologica 100, 479-488 (2015).

13. Cervantes, F. et al. Three-year efficacy, safety, and survival findings from COMFORT-II, a phase 3 study comparing ruxolitinib with best available therapy for myelofibrosis. Blood 122, 4047-4053 (2013).

14. Verstovsek, S. et al. Long-term treatment with ruxolitinib for patients with myelofibrosis: 5-year update from the randomized, double-blind, placebocontrolled, phase 3 COMFORT-I trial. J. Hematol. Oncol. 10, 55 (2017).
15. Harrison, C. N. et al. Long-term findings from COMFORT-II, a phase 3 study of ruxolitinib vs best available therapy for myelofibrosis. Leukemia 30, 1701-1707 (2016).

16. Newbern, K. J. et al. Clonal evolution and outcomes in myelofibrosis after ruxolitinib discontinuation. Blood 130, 1125-1131 (2017).

17. Pardanani, A. \& Tefferi, A. How I treat myelofibrosis after failure of JAK inhibitors. Blood 132, 492-500 (2018).

18. Meyer, S. C. Mechanisms of Resistance to JAK2 Inhibitors in Myeloproliferative Neoplasms. Hematol. Oncol. Clin. North. Am. 31, 627-642 (2017).

19. Koppikar, P. et al. Heterodimeric JAK-STAT activation as a mechanism of persistence to JAK2 inhibitor therapy. Nature 489, 155-159 (2012).

20. Kalota, A., Jeschke, G. R., Carroll, M. \& Hexner, E. O. Intrinsic Resistance to JAK2 Inhibition in Myelofibrosis. Clin. Cancer Res. 19, 1729-1739 (2013).

21. Meyer Sara, C. et al. CHZ868, a Type II JAK2 Inhibitor, Reverses Type I JAK Inhibitor Persistence and Demonstrates Efficacy in Myeloproliferative Neoplasms. Cancer Cell. 28, 15-28 (2015).

22. Deshpande, A. et al. Kinase domain mutations confer resistance to novel inhibitors targeting JAK2V617F in myeloproliferative neoplasms. Leukemia $\mathbf{2 6}$, 708-715 (2012)

23. Weigert, $\mathrm{O}$. et al. Genetic resistance to JAK2 enzymatic inhibitors is overcome by HSP90 inhibition. J. Exp. Med. 209, 259-273 (2012).

24. Guglielmelli, P. et al. Impact of mutational status on outcomes in myelofibrosis patients treated with ruxolitinib in the COMFORT-II Study. Blood 123, 2157-2160 (2014)

25. Barosi, G. et al. JAK2(V617F) allele burden $50 \%$ is associated with response to ruxolitinib in persons with MPN-associated myelofibrosis and splenomegaly requiring therapy. Leukemia 30, 1772-1775 (2016).

26. Patel, K. P. et al. Correlation of mutation profile and response in patients with myelofibrosis treated with ruxolitinib. Blood 126, 790-797 (2015).

27. Spiegel, J. Y. et al. Impact of genomic alterations on outcomes in myelofibrosis patients undergoing JAK1/2 inhibitor therapy. Blood Adv. 1, 1729-1738 (2017)

28. Barbui, T., Thiele, J., Vannucchi, A. M. \& Tefferi, A. Rationale for revision and proposed changes of the WHO diagnostic criteria for polycythemia vera, essential thrombocythemia and primary myelofibrosis. Blood Cancer J. 5, e337 (2015).

29. Swerdlow S. H., et al. WHO Classification of Tumours of Haematopoietic and Lymphoid Tissues. (IARC, Lyon, 2017).

30. Barosi, G. et al. Proposed criteria for the diagnosis of post-polycythemia vera and post-essential thrombocythemia myelofibrosis: a consensus statement from the international working group for myelofibrosis research and treatment. Leukemia 22, 437-438 (2008).

31. Tefferi, A. et al. Revised response criteria for myelofibrosis: Internationa Working Group-Myeloproliferative Neoplasms Research and Treatment (IWGMRT) and European LeukemiaNet (ELN) consensus report. Blood 122 1395-1398 (2013).

32. Emanuel, R. M. et al. Myeloproliferative neoplasm (MPN) symptom assessment form total symptom score: prospective international assessment of an abbreviated symptom burden scoring system among patients with MPNs. J. Clin. Oncol. 30, 4098-40103 (2012).

33. Lippert, E. et al. The JAK2-V617F mutation is frequently present at diagnosis in patients with essential thrombocythemia and polycythemia vera. Blood $\mathbf{1 0 8}$ 1865-1867 (2006).

34. Pancrazzi, A. et al. A sensitive detection method for MPLW515L or MPLW515K mutation in chronic myeloproliferative disorders with locked nucleic acidmodified probes and real-time polymerase chain reaction. J. Mol. Diagn. 10, 435-441 (2008).

35. Pietra, D. et al. Deep sequencing reveals double mutations in cis of MPL exon 10 in myeloproliferative neoplasms. Haematologica 96, 607-611 (2011).

36. Guglielmelli, P. et al. Anaemia characterises patients with myelofibrosis harbouring Mpl mutation. Br. J. Haematol. 137, 244-247 (2007).

37. Tefferi, A. et al. CALR vs JAK2 vs MPL mutated or triple-negative myelofibrosis: clinical, cytogenetic and molecular comparisons. Leukemia 28, 1472-1477 (2014).

38. Guglielmelli, P. et al. Validation of the differential prognostic impact of type 1/ type 1-like versus type 2/type 2-like CALR mutations in myelofibrosis. Blood Cancer I. 5, e360 (2015).

39. Verstovsek, S. et al. The clinical benefit of ruxolitinib across patient subgroups: analysis of a placebocontrolled, Phase III study in patients with myelofibrosis. Br. J. Haematol. 161, 508-516 (2013).

40. Guglielmelli, P. et al. Ruxolitinib is an effective treatment for CALR-positive patients with myelofibrosis. Br. J. Haematol. 173, 938-940 (2015). 
41. Porpaczy, E. et al. Aggressive B-cell lymphomas in patients with myelofibrosis receiving JAK1/2 inhibitor therapy. Blood 132, 694-706 (2018).

42. Vannucchi, A. M. et al. Ruxolitinib reduces JAK2p.V617F allele burden in patients with polycythemia vera enrolled in the RESPONSE study. Ann. Hematol. 96, 1113-1120 (2017).

43. Pieri, L. et al. JAK2V617F complete molecular remission in polycythemia vera/ essential thrombocythemia patients treated with ruxolitinib. Blood 125, 3352-3353 (2015).

44. Vainchenker, W. \& Kralovics, R. Genetic basis and molecular pathophysiology of classical myeloproliferative neoplasms. Blood 129, 3146-3158 (2017).

45. Plo, I. et al. JAK2 stimulates homologous recombination and genetic instability: potential implication in the heterogeneity of myeloproliferative disorders. Blood 112, 1402-1412 (2008).
46. Dawson, M. A. et al. JAK2 phosphorylates histone H3Y41 and excludes HP1alpha from chromatin. Nature 461, 819-822 (2009).

47. Zhao, J., Sun, B. K., Erwin, J. A., Song, J. J. \& Lee, J. T. Polycomb proteins targeted by a short repeat RNA to the mouse X chromosome. Science $\mathbf{3 2 2}$, 750-756 (2008).

48. Ahn, J. S. et al. JAK2V617F mediates resistance to DNA damage-induced apoptosis by modulating FOXO3A localization and $\mathrm{BCl}-\mathrm{xL}$ deamidation. Oncogene 35, 22353-2246 (2016).

49. Marty, C. et al. A role for reactive oxygen species in JAK2 V617F myeloproliferative neoplasm progression. Leukemia 27, 2187-2195 (2013).

50. Vannucchi, A. M. \& Guglielmelli, P. Traffic lights for ruxolitinib. Blood 130, 1075-1076 (2017). 